\title{
La Station Biologique de Roscoff
}

Fédération de recherche du CNRS, École Interne de l'Université Pierre et Marie Curie, Observatoire océanologique de l'Institut National des Sciences de l'Univers (INSU)

Créée en 1872 par Henri De Lacaze-Duthiers, Professeur à la Sorbonne, la Station Biologique de Roscoff (SBR) est un centre de recherche et d'enseignement en biologie marine et en océanologie situé sur la côte nord de la Bretagne, au bord d'une mer à fortes marées.

Aujourd'hui rattachée à l'Université Pierre et Marie Curie, au CNRS et à l'INSU, la SBR est un acteur majeur de la recherche en biologie marine au niveau européen et international. 220 chercheurs, enseignantschercheurs, ingénieurs, techniciens, doctorants et postdoctorants y travaillent.

\section{Les missions de la Station Biologique de Roscoff}

- promouvoir la recherche et l'enseignement de la biologie des organismes marins et des écosystèmes;

- donner accès aux ressources écologiques et biologiques du site pour la communauté scientifique nationale et internationale;

- observer à long terme l'évolution des caractéristiques physico-chimiques et biologiques de l'environnement côtier;

- assurer l'accueil scientifique et pédagogique.

Les recherches menées à la Station Biologique de Roscoff abordent des domaines d'étude qui vont de la structure fine et du fonctionnement de la macromolécule biologique à celui de l'océan global.

\section{Principales thématiques de recherche}

- l'étude des organismes marins et des écosystèmes;

- la compréhension des processus d'adaptation et d'évolution de ces organismes;

- la recherche de molécules actives (enzymes et produits naturels) pour des applications médicales par exemple.

Ces travaux concernent plus particulièrement l'étude du cycle et de la physiologie cellulaires, la biochimie et le développement des algues brunes et rouges, l'écophysiologie de la faune hydrothermale, la diversité du phytoplancton et du zooplancton, l'évolution et la génétique des populations, l'écologie benthique. En chimie marine des recherches sont menées sur les traceurs chimiques pour comprendre les phénomènes de circulation océanique des masses d'eau. Un très fort accent est mis sur les approches de biologie systémique.

\section{Chiffres-clés de la recherche}

Une fédération de recherche regroupant quatre unités de recherche (CNRS/Université Pierre et Marie Curie).
100 publications par an dans les revues scientifiques internationales.

8 à 10 conférences internationales organisées par an (incluant les Conférences Monod et les Conférences Gordon).

24 brevets déposés (depuis 2000).

23 thèses de doctorats complétées en 2005/2006.

\section{Principaux réseaux et programmes de collaboration}

Au niveau national

- Membre fondateur du pôle de compétitivité «Mer» Bretagne reconnu par l'État comme l'un des pôles de compétitivité à vocation mondiale.

- Membre fondateur de l'Europôle Mer, pôle de recherche européen regroupant les principaux acteurs de Bretagne Occidentale en matière de recherche, de formation, de valorisation et de diffusion scientifique dans le domaine des sciences et techniques de la mer.

- Membre du Cancéropôle Grand Ouest, qui rassemble les établissements de recherche sur le cancer dans le Grand Ouest.

- Membre fondateur de OUEST génopole ${ }^{\circledR}$, qui œuvre pour le développement de la génomique et de la postgénomique en Bretagne et dans les Pays de la Loire.

Au niveau international

- Coordinateur du réseau d'excellence européen Marine Genomics Europe : ce réseau rassemble 45 institutions de recherche dans toute l'Europe.

Objectif : renforcer les synergies européennes et développer les recherches basées sur les approches de génomique pour comprendre le fonctionnement des écosystèmes et la biologie des organismes marins.

- Partenaire de l’Université Pontificale du Chili dans le cadre du Laboratoire International Associé "Dispersal and Adaptation in Marine Species (DIAMS)".

Objectifs du programme : étudier les processus biologiques affectant la distribution et la diversité des espèces et des populations ; analyser l'influence sur ces processus des changements climatiques et de l'activité humaine dans les eaux marines côtières.

- Partenaire des réseaux d'excellence européens :

Marbef (93 organismes de recherche partenaires).Thématique : biodiversité marine.

Eur-Océans (66 organismes de recherche partenaires). Thématiques : changements globaux et fonctionnement des écosystèmes marins pélagiques. 


\section{L'observation du milieu côtier}

Reconnue Observatoire des Sciences de l'Univers (OSU) depuis 1985 par l'Institut National des Sciences de l'Univers (INSU), la Station Biologique de Roscoff réalise une mission d'observation du littoral dans le cadre des réseaux nationaux suivants :

- le Service d'Observation du Milieu Littoral (SOMLIT). Ce service regroupe sept stations marines. Il réalise des séries d'observations à long terme sur les côtes de la Manche, de l'Atlantique et de la Méditerranée pour différents paramètres hydro-climatiques afin de mieux comprendre l'impact des changements climatiques et des activités humaines sur le fonctionnement des écosystèmes;

- le réseau REBENT. Coordonné par l'Ifremer, il est dédié à la surveillance des écosystèmes benthiques. Les études fournissent des données références pour évaluer les changements naturels ou induits par l'activité humaine.

\section{La formation à et par la recherche}

La Station Biologique de Roscoff est l'un des trois sites de formation en science de la mer délocalisés de l'Université Pierre et Marie Curie (Paris VI). Les étudiants de master viennent suivre sur le campus de Roscoff des unités d'enseignement dans les domaines suivants : biologie cellulaire, génétique, sciences du végétal, physiologie, écologie marine, biodiversité.
La Station Biologique de Roscoff a par ailleurs été sélectionnée dans le cadre des actions «Marie Curie» initiées par la Commission Européenne pour donner la possibilité à de jeunes scientifiques de talent de réaliser leur thèse dans un pays d'Europe au sein d'équipes de recherche internationales. La SBR assure ainsi la formation de onze jeunes chercheurs de toutes nationalités. Le programme mis en place à Roscoff offre une formation d'excellence à la recherche dans le domaine de la biologie marine et de ses technologies émergentes.

\section{Un centre de conférence pour les colloques scientifiques}

La Station Biologique de Roscoff a une longue tradition d'accueil de chercheurs, d'enseignants et d'étudiants. Le centre de conférence labellisé par le CNRS offre tous les services et la capacité hôtelière nécessaires à l'organisation de colloques scientifiques : salle de conférence d'une centaine de places, salles de réunion, restauration, location de chambres ou studios.

Station biologique de Roscoff place Georges Teissier

BP 74, 29682 Roscoff cedex, France Tél. : + 33298292323

Fax : +33298292324-www.wb-roscoff.fr 